\title{
A Characterization of Bi-Jordan Homomorphisms on Banach Algebras
}

\begin{abstract}
Abbas Zivari-Kazempour
Department of Mathematics, Ayatollah Borujerdi University, Borujerd, Iran

Correspondence should be addressed to Abbas Zivari-Kazempour; zivari@abru.ac.ir

Received 6 November 2016; Revised 2 January 2017; Accepted 16 January 2017; Published 31 January 2017

Academic Editor: Baruch Cahlon

Copyright ( 2017 Abbas Zivari-Kazempour. This is an open access article distributed under the Creative Commons Attribution License, which permits unrestricted use, distribution, and reproduction in any medium, provided the original work is properly cited.
\end{abstract}

For Banach algebras $\mathscr{A}$ and $\mathscr{B}$, we show that if $\mathcal{U}=\mathscr{A} \times \mathscr{B}$ is unital and commutative, each bi-Jordan homomorphism from $\mathscr{U}$ into a semisimple commutative Banach algebra $\mathscr{D}$ is a bihomomorphism.

\section{Introduction}

Let $\mathscr{A}$ and $\mathscr{B}$ be complex Banach algebras and let $\varphi: \mathscr{A} \rightarrow \mathscr{B}$ be a linear map. Then $\varphi$ is called $n$-homomorphism if for all $a_{1}, a_{2}, \ldots, a_{n} \in \mathscr{A}$

$$
\varphi\left(a_{1} a_{2} \cdots a_{n}\right)=\varphi\left(a_{1}\right) \varphi\left(a_{2}\right) \cdots \varphi\left(a_{n}\right) .
$$

The concept of $n$-homomorphism was studied for complex algebras by Hejazian et al. in [1]. A 2-homomorphism is then just a homomorphism, in the usual sense. We refer the reader to [2], for certain properties of 3-homomorphisms.

In [3], Eshaghi Gordji introduced the concept of an $n$ Jordan homomorphism. A linear map $\varphi$ between Banach algebras $\mathscr{A}$ and $\mathscr{B}$ is called an $n$-Jordan homomorphism if

$$
\varphi\left(a^{n}\right)=\varphi(a)^{n}, \quad(a \in \mathscr{A}) .
$$

A 2-Jordan homomorphism is called simply a Jordan homomorphism.

It is obvious that each $n$-homomorphism is an $n$-Jordan homomorphism, but in general the converse is false. The converse statement may be true under certain conditions. For example, Zelazko in [4] proved that every Jordan homomorphism from Banach algebra $\mathscr{A}$ into a semisimple commutative Banach algebra $\mathscr{B}$ is a homomorphism. See also [5] for another approach to the same result. The reader is referred to [6], for characterization of 3-Jordan homomorphism.

Also it is shown in [3] that every $n$-Jordan homomorphism between two commutative Banach algebras is an $n$ homomorphism for $n \in\{2,3,4\}$ and this result is extended to the case $n=5$ in [7]. Some investigation has been done about Jordan derivations and Jordan centralizers in $[8,9]$.

Throughout the paper, let $\mathscr{U}=\mathscr{A} \times \mathscr{B}$. Then $\mathcal{U}$ is a Banach algebra for the multiplication

$$
(a, b)(x, y)=(a x, b y), \quad(a, b),(x, y) \in \mathcal{U},
$$

and with norm

$$
\|(a, b)\|=\|a\|+\|b\| .
$$

Clearly, $\mathcal{U}$ is commutative if and only if both $\mathscr{A}$ and $\mathscr{B}$ are commutative, and it is unital if and only if both $\mathscr{A}$ and $\mathscr{B}$ are unital. Without any confusion we denote by $e$, the unit element of both $\mathscr{A}$ and $\mathscr{B}$.

Let $\mathscr{D}$ be a complex Banach algebra. A bilinear map is a function $\varphi: \mathscr{U} \rightarrow \mathscr{D}$ such that for any $a \in \mathscr{A}$ the map $b \mapsto$ $\varphi(a, b)$ is linear map from $\mathscr{B}$ to $\mathscr{D}$, and for any $b \in \mathscr{B}$ the map $a \mapsto \varphi(a, b)$ is linear map from $\mathscr{A}$ to $\mathscr{D}$.

A bilinear map $\varphi$ is called bihomomorphism if for all $(a, b),(x, y) \in \mathscr{U}$,

$$
\varphi(a x, b y)=\varphi(a, b) \varphi(x, y),
$$

and it is called bi-Jordan homomorphism (BJH, for short) if

$$
\varphi\left(a^{2}, b^{2}\right)=\varphi(a, b)^{2}, \quad(a, b) \in \mathcal{U} .
$$


It is obvious that each bihomomorphism is $\mathrm{BJH}$, but in general the converse is not true. For example, let

$$
\mathscr{A}=\left\{\left[\begin{array}{ll}
a & b \\
0 & 0
\end{array}\right]: a, b \in \mathbb{R}\right\},
$$

and $\mathscr{B}=\mathscr{A} \cup\{I\}$, where $I=\left[\begin{array}{ll}1 & 0 \\ 0 & 1\end{array}\right]$. Define $\varphi: \mathscr{A} \times \mathscr{B} \rightarrow \mathscr{A}$ by $\varphi(x, y)=x y$. Then $\varphi$ is bilinear and

$$
\varphi\left(x^{2}, y^{2}\right)=x^{2} y^{2}=(x y)^{2}=\varphi(x, y)^{2},
$$

for all $(x, y) \in \mathscr{U}=\mathscr{A} \times \mathscr{B}$. Thus, $\varphi$ is a bi-Jordan homomorphism, but it is not bihomomorphism. For instance, let

$$
\begin{aligned}
& u=\left[\begin{array}{ll}
a & b \\
0 & 0
\end{array}\right], \\
& x=\left[\begin{array}{ll}
c & d \\
0 & 0
\end{array}\right], \\
& v=\left[\begin{array}{ll}
s & t \\
0 & 0
\end{array}\right], \\
& y=I .
\end{aligned}
$$

Then $(u, v),(x, y) \in \mathscr{U}$ and

$$
\begin{aligned}
\varphi(u x, v y) & =\left[\begin{array}{cc}
a c s & a c t \\
0 & 0
\end{array}\right] \neq\left[\begin{array}{cc}
a s c & a s d \\
0 & 0
\end{array}\right] \\
& =\varphi(u, v) \varphi(x, y) .
\end{aligned}
$$

In this paper, we show that the converse statement holds under certain conditions.

\section{Main Results}

We commence with some useful lemmas.

Lemma 1. Suppose that $\varphi: \mathscr{U} \rightarrow \mathbb{C}$ is a BJH. Then

(1) $\varphi\left(x y+y x, b^{2}\right)=2 \varphi(x, b) \varphi(y, b)$;

(2) $\varphi\left(a^{2}, x y+y x\right)=2 \varphi(a, x) \varphi(a, y)$.

Proof. The proof is straightforward.

Lemma 2. Suppose that $\varphi: \mathscr{U} \rightarrow \mathbb{C}$ is a BJH. If $\mathscr{U}$ is unital and commutative, then

(1) $\varphi(a x, e)=\varphi(a, e) \varphi(x, e)$;

(2) $\varphi(e, b y)=\varphi(e, b) \varphi(e, y)$.

Proof. The proof follows from Lemma 1.

Lemma 3. Let $\mathscr{U}$ be unital, and let $\varphi: \mathscr{U} \rightarrow \mathbb{C}$ be a nonzero $B J H$. Then $\varphi(e, e) \neq 0$.

Proof. Let $\varphi$ be a BJH. Then for all $(a, b) \in \mathcal{U}$, we get

$$
\varphi\left(a^{2}, b^{2}\right)=\varphi(a, b)^{2} .
$$

Replacing $a$ by $x+e$ and $b$ by $y+e$ in (11) gives

$$
\varphi\left(x^{2}+2 x+e, y^{2}+2 y+e\right)=\varphi(x+e, y+e)^{2} .
$$

Assume that $\varphi(e, e)=0$; then by Lemma 1,

$$
\varphi(x, e)=\varphi(e, y)=0, \quad(x, y) \in \mathcal{U} .
$$

It follows from (12) and (13) that

$$
\varphi(2 x, 2 y)+\varphi\left(2 x, y^{2}\right)+\varphi\left(x^{2}, 2 y\right)=0
$$

for all $(x, y) \in \mathscr{U}$. By Lemma 1 ,

$$
\begin{aligned}
& \varphi\left(2 x, y^{2}\right)=2 \varphi(x, y) \varphi(e, y)=0 \\
& \varphi\left(x^{2}, 2 y\right)=2 \varphi(x, e) \varphi(x, y)=0 .
\end{aligned}
$$

Thus, by (14) and (15), we get $\varphi(x, y)=0$, for all $(x, y) \in \mathcal{U}$, which is contradiction.

Now we state and prove the main theorem. The main idea of the proof can be found in [6].

Theorem 4. Let $\mathcal{U}$ be unital and commutative, and let $\varphi$ be a BJH from $\mathcal{U}$ into a semisimple commutative Banach algebra D. Then $\varphi$ is a bihomomorphism.

Proof. We first assume that $\mathscr{D}=\mathbb{C}$ and let $\varphi: \mathcal{U} \rightarrow \mathbb{C}$ be a $\mathrm{BJH}$. Then, for all $(a, b) \in \mathcal{U}$, we get

$$
\varphi\left(a^{2}, b^{2}\right)=\varphi(a, b)^{2} .
$$

Replacing $a$ by $x+e$ and $b$ by $y+e$ in (16), we have

$$
\varphi\left(x^{2}+2 x+e, y^{2}+2 y+e\right)=\varphi(x+e, y+e)^{2} .
$$

By Lemma 3, $\varphi(e, e) \neq 0$, so (16) gives

$$
\varphi(e, e)=1 \text {. }
$$

It follows from Lemma 1 that

$$
\begin{aligned}
& \varphi\left(2 x, y^{2}\right)=2 \varphi(x, y) \varphi(e, y), \\
& \varphi\left(x^{2}, 2 y\right)=2 \varphi(x, e) \varphi(x, y) .
\end{aligned}
$$

Thus, by (17), (18), and (19) we get

$$
\varphi(x, y)=\varphi(x, e) \varphi(e, y)
$$

for all $(x, y) \in \mathcal{U}$. Replacing $x$ by ax and $y$ by by in (20) gives

$$
\varphi(a x, b y)=\varphi(a x, e) \varphi(e, b y) .
$$

By (20) and Lemma 2 we deduce

$$
\begin{aligned}
\varphi(a, b) \varphi(x, y) & =[\varphi(a, e) \varphi(x, e)][\varphi(e, b) \varphi(e, y)] \\
& =\varphi(a x, e) \varphi(e, b y) .
\end{aligned}
$$


By (21) and (22),

$$
\varphi(a x, b y)=\varphi(a, b) \varphi(x, y)
$$

for all $(a, b),(x, y) \in \mathcal{U}$, and so $\varphi$ is bihomomorphism.

Now suppose that $\mathscr{D}$ is semisimple and commutative. Let $\mathfrak{M}(\mathscr{D})$ be the maximal ideal space of $\mathscr{D}$. We associate with each $f \in \mathfrak{M}(\mathscr{D})$ a function $\varphi_{f}: \mathcal{U} \rightarrow \mathbb{C}$ defined by

$$
\varphi_{f}(a, b):=f(\varphi(a, b)), \quad(a, b) \in \mathcal{U}
$$

Pick $f \in \mathfrak{M}(\mathscr{D})$ arbitrary. It is easy to see that $\varphi_{f}$ is a $\mathrm{BJH}$, so by the above argument it is a bihomomorphism. Thus by the definition of $\varphi_{f}$ we have

$$
\begin{aligned}
f(\varphi(a x, b y)) & =f(\varphi(a, b)) f(\varphi(x, y)) \\
& =f(\varphi(a, b) \varphi(x, y)) .
\end{aligned}
$$

Since $f \in \mathfrak{M}(\mathscr{D})$ was arbitrary and $\mathscr{D}$ is assumed to be semisimple, we obtain

$$
\varphi(a x, b y)=\varphi(a, b) \varphi(x, y)
$$

for all $(a, b),(x, y) \in \mathcal{U}$.

The second dual space $\mathscr{A}^{\prime \prime}$ of a Banach algebra $\mathscr{A}$ admits two Banach algebra multiplications known as the first and second Arens products, each extending the product on $\mathscr{A}$. These products which we denote by $\square$ and $\diamond$, respectively, can be defined as follows:

$$
\begin{aligned}
& F \square G=w^{*}-\lim _{i} \lim _{j} a_{i} b_{j}, \\
& F \diamond G=w^{*}-\lim _{j} \lim _{i} a_{i} b_{j},
\end{aligned}
$$

where $\left(a_{i}\right)$ and $\left(b_{j}\right)$ are nets in $\mathscr{A}$ that converge, in $w^{*}$ topologies, to $F$ and $G$, respectively. The Banach algebra $\mathscr{A}$ is said to be Arens regular if $F \square G=F \diamond G$ on the whole of $\mathscr{A}^{\prime \prime}$. Some significant results related to the Arens regularity of certain bilinear maps and Banach algebra obtained in [10]. For more information on the Arens products, we refer the reader to $[11,12]$, for example.

It is shown in [11] that every $C^{*}$-algebra $\mathscr{A}$ is Arens regular and semisimple. Also the second dual of each $C^{*}$-algebra is also a $C^{*}$-algebra.

Theorem 5. If $k$ is the natural embedding of a Banach space $X$ into $X^{\prime \prime}$, then $k(X)$ is $w^{*}$-dense in $X^{\prime \prime}$.

Proof. See [13].

Theorem 6. Let $\varphi: \mathscr{U} \rightarrow \mathscr{D}$ be a continuous BJH. Then the second adjoint $\varphi^{\prime \prime}: \mathcal{U}^{\prime \prime} \rightarrow \mathscr{D}^{\prime \prime}$ of $\varphi$ is also a BJH.
Proof. By Theorem 5, there are bounded nets $\left(a_{i}\right)$ and $\left(b_{j}\right)$ in $\mathscr{A}$ and $\mathscr{B}$ that converge, in $w^{*}$-topologies, to $F$ and $G$, respectively. Then

$$
\begin{aligned}
\varphi^{\prime \prime}\left(F^{2}, G^{2}\right) & =\varphi^{\prime \prime}\left(w^{*}-\lim _{i} a_{i}^{2}, w^{*}-\lim _{j} b_{j}^{2}\right) \\
& =\varphi\left(w^{*}-\lim _{i} a_{i}^{2}, w^{*}-\lim _{j} b_{j}^{2}\right) \\
& =w^{*}-\lim _{i} \lim _{j} \varphi\left(a_{i}^{2}, b_{j}^{2}\right) \\
& =w^{*}-\lim _{i} \lim _{j} \varphi\left(a_{i}, b_{j}\right)^{2} \\
& =w^{*}-\lim _{i} \lim _{j} \varphi^{\prime \prime}\left(a_{i}, b_{j}\right)^{2} \\
& =\varphi^{\prime \prime}\left(w^{*}-\lim _{i} a_{i}, w^{*}-\lim _{j} b_{j}\right)^{2} \\
& =\varphi^{\prime \prime}(F, G)^{2} .
\end{aligned}
$$

Thus, $\varphi^{\prime \prime}$ is BJH, as claimed.

Since the second dual of every $C^{*}$-algebra is unital [14], we deduce the following result from Theorems 4 and 6 .

Corollary 7. Let $\varphi: \mathscr{U} \rightarrow \mathscr{D}$ be a continuous BJH between commutative $C^{*}$-algebras. Then $\varphi^{\prime \prime}: \mathcal{U}^{\prime \prime} \rightarrow \mathscr{D}^{\prime \prime}$ is a bihomomorphism.

Theorem 8. Suppose that $\varphi$ is a BJH from the unital Banach algebra $\mathcal{U}$ into $\mathbb{C}$. Then $\varphi$ is a $n-B J H$; that is,

$$
\varphi\left(a^{n}, b^{n}\right)=\varphi(a, b)^{n}
$$

for all $n \geq 3$.

Proof. Let $\varphi: \mathcal{U} \rightarrow \mathbb{C}$ be a BJH. Then, for all $(a, b) \in \mathcal{U}$,

$$
\varphi\left(a^{2}, b^{2}\right)=\varphi(a, b)^{2} \text {. }
$$

Replacing $a$ by $a+x$ and $b$ by $b+y$ in (30), we have

$$
\begin{aligned}
\varphi(a x+x a, b y+y b)= & 2 \varphi(a, b) \varphi(x, y) \\
& +2 \varphi(a, y) \varphi(x, b) .
\end{aligned}
$$

Replacing $a$ by $a^{2}$ and $b$ by $b^{2}$ in (31) gives

$$
\begin{aligned}
\varphi & \left(a^{2} x+x a^{2}, b^{2} y+y b^{2}\right) \\
& =2 \varphi\left(a^{2}, b^{2}\right) \varphi(x, y)+2 \varphi\left(a^{2}, y\right) \varphi\left(x, b^{2}\right) .
\end{aligned}
$$

Replacing $x$ by $a$ and $y$ by $b$ in (32), we obtain

$$
\begin{aligned}
\varphi\left(2 a^{3}, 2 b^{3}\right)= & 2 \varphi\left(a^{2}, b^{2}\right) \varphi(a, b) \\
& +2 \varphi\left(a^{2}, b\right) \varphi\left(a, b^{2}\right) .
\end{aligned}
$$


Replacing $x$ and $y$ by $e$ in (31), we get

$$
\varphi(2 a, 2 b)=2 \varphi(a, b) \varphi(e, e)+2 \varphi(a, e) \varphi(e, b) .
$$

Since $\varphi(e, e)=1$, so (34) gives

$$
\varphi(a, b)=\varphi(a, e) \varphi(e, b),
$$

for all $(a, b) \in \mathcal{U}$. By Lemma 1 ,

$$
\begin{aligned}
& \varphi\left(a^{2}, b\right)=\varphi(a, b) \varphi(a, e), \\
& \varphi\left(a, b^{2}\right)=\varphi(a, b) \varphi(e, b) .
\end{aligned}
$$

It follows from (35) and (36) that

$$
\begin{aligned}
\varphi\left(a^{2}, b\right) \varphi\left(a, b^{2}\right) & =\varphi(a, b)^{2} \varphi(a, e) \varphi(e, b) \\
& =\varphi(a, b)^{3} .
\end{aligned}
$$

By (33) and (37),

$$
\varphi\left(a^{3}, b^{3}\right)=\varphi(a, b)^{3},
$$

for all $(a, b) \in \mathcal{U}$. Thus, the result is established for $n=3$. An easy induction argument now finishes the proof.

As a consequence of Theorem 8 we have the next result.

Corollary 9. Suppose that $\varphi$ is a BJH from the unital Banach algebra $\mathcal{U}$ into a semisimple commutative Banach algebra $\mathscr{D}$. Then $\varphi$ is an $n-B J H$.

Baker in [15] proved that an almost multiplicative function is either bounded or multiplicative. Now we prove an analogous result of Baker's theorem for bihomomorphism.

Theorem 10. Let $\delta>0$ and $\varphi: \mathscr{U} \rightarrow \mathbb{C}$ satisfy

$$
|\varphi(a x, b y)-\varphi(a, b) \varphi(x, y)| \leq \delta,
$$

for all $(a, b),(x, y) \in \mathcal{U}$. Then either $\varphi$ is bihomomorphism or

$$
|\varphi(x, y)| \leq \frac{1}{2}(1+\sqrt{1+4 \delta}):=\varepsilon,
$$

for all $(x, y) \in \mathcal{U}$.

Proof. Let $\varepsilon^{2}-\varepsilon=\delta$ and $\varepsilon>1$. Suppose that there exist $(s, t) \in \mathcal{U}$ such that $|\varphi(s, t)|>\varepsilon$, so that $|\varphi(s, t)|=\varepsilon+p$, where $p>0$. Then we have

$$
\begin{aligned}
\left|\varphi\left(s^{2}, t^{2}\right)\right| & \geq\left|\varphi(s, t)^{2}-\left(\varphi(s, t)^{2}-\varphi\left(s^{2}, t^{2}\right)\right)\right| \\
& \geq|\varphi(s, t)|^{2}-\left|\varphi(s, t)^{2}-\varphi\left(s^{2}, t^{2}\right)\right| ;
\end{aligned}
$$

hence

$$
\begin{aligned}
\left|\varphi\left(s^{2}, t^{2}\right)\right| & \geq|\varphi(s, t)|^{2}-\delta=(\varepsilon+p)^{2}-\delta \\
& =2 \varepsilon p+p^{2}+\varepsilon>\varepsilon+2 p .
\end{aligned}
$$

Now make the induction assumption:

$$
\left|\varphi\left(s^{2^{n}}, t^{2^{n}}\right)\right|>\varepsilon+(n+1) p .
$$

Let

$$
M_{n}=\varphi\left(s^{2^{n}}, t^{2^{n}}\right) .
$$

Then

$$
\begin{aligned}
\left|M_{n+1}\right| & =\left|M_{n}^{2}-\left(M_{n}^{2}-M_{n+1}\right)\right| \geq\left|M_{n}^{2}\right|-\delta \\
& =\left|M_{n}\right|^{2}+\varepsilon-\varepsilon^{2},
\end{aligned}
$$

whence

$$
\begin{aligned}
\left|M_{n+1}\right| & \geq(\varepsilon+(n+1) p)^{2}+\varepsilon-\varepsilon^{2} \\
& =(2 n+2) \varepsilon p+\varepsilon+(n+1)^{2} p^{2} \\
& >\varepsilon+(n+2) p,
\end{aligned}
$$

and (43) is established for all $n \in \mathbb{N}$.

Given that $(a, b),(x, y),(u, v) \in \mathcal{U}$, we have

$$
\begin{gathered}
|\varphi(a x u, b y v)-\varphi(a, b) \varphi(x u, y v)| \leq \delta, \\
|\varphi(a x u, b y v)-\varphi(a x, b y) \varphi(u, v)| \leq \delta .
\end{gathered}
$$

Thus,

$$
|\varphi(a x, b y) \varphi(u, v)-\varphi(a, b) \varphi(x u, y v)| \leq 2 \delta .
$$

Hence

$$
\begin{aligned}
|\varphi(a x, b y) \varphi(u, v)-\varphi(a, b) \varphi(x, y) \varphi(u, v)| \\
\leq|\varphi(a x, b y) \varphi(u, v)-\varphi(a, b) \varphi(x u, y v)| \\
\quad+|\varphi(a, b) \varphi(x u, y v)-\varphi(a, b) \varphi(x, y) \varphi(u, v)| \\
\leq 2 \delta+\delta|\varphi(a, b)| .
\end{aligned}
$$

Therefore

$$
\begin{aligned}
& |\varphi(u, v)||\varphi(a x, b y)-\varphi(a, b) \varphi(x, y)| \\
& \quad \leq 2 \delta+\delta|\varphi(a, b)| .
\end{aligned}
$$

Now put $(u, v)=\left(s^{2^{n}}, t^{2^{n}}\right)$ to obtain

$$
\begin{aligned}
& |\varphi(a x, b y)-\varphi(a, b) \varphi(x, y)| \leq \frac{2 \delta+\delta|\varphi(a, b)|}{\left|M_{n}\right|} \\
& \quad \leq \frac{2 \delta+\delta|\varphi(a, b)|}{\varepsilon+(n+1) p}
\end{aligned}
$$

for all $n \in \mathbb{N}$ and $(a, b) \in \mathcal{U}$. Letting $n \rightarrow \infty$, we get $\varphi(a x, b y)=\varphi(a, b) \varphi(x, y)$ and so $\varphi$ is a bihomomorphism. 
As Baker pointed out in his article, the above proof works for functions $\varphi: \mathscr{U} \rightarrow \mathscr{D}$, where $\mathscr{D}$ is a Banach algebra in which the norm is multiplicative; that is, $\|x y\|=\|x\|\|y\|$ for all $x, y \in \mathscr{D}$. For norm algebra $\mathscr{D}$ for which the norm is not multiplicative, the situation is false. For example, let $\delta>0$ and choose $\varepsilon>0$ so that $\left|\varepsilon-\varepsilon^{2}\right|=\delta$, and define $\varphi: \mathbb{R} \times \mathbb{R} \rightarrow$ $M_{3}(\mathbb{R})$ by

$$
\varphi(x, y)=\left[\begin{array}{lll}
x & 0 & 0 \\
0 & y & 0 \\
0 & 0 & \varepsilon
\end{array}\right] \quad x, y \in \mathbb{R}
$$

Then with the usual matrix norm

$$
\|\varphi(a x, b y)-\varphi(a, b) \varphi(x, y)\|=\delta,
$$

for all $(a, b),(x, y) \in \mathbb{R} \times \mathbb{R}$. Clearly, $\varphi$ is unbounded, but $\varphi$ is not bihomomorphism.

\section{Competing Interests}

The author declares that there is no conflict of interests regarding the publication of this paper.

\section{References}

[1] Sh. Hejazian, M. Mirzavaziri, and M. S. Moslehian, "nhomomorphisms," Bulletin of the Iranian Mathematical Society, vol. 31, no. 1, pp. 13-23, 2005.

[2] J. Braĉîc and M. S. Moslehian, "On automatic continuity of 3homomorphisms on Banach algebras," Bulletin of the Malaysian Mathematical Sciences Society. Second Series, vol. 30, no. 2, pp. 195-200, 2007.

[3] M. Eshaghi Gordji, " $n$-Jordan homomorphisms," Bulletin of the Australian Mathematical Society, vol. 80, no. 1, pp. 159-164, 2009.

[4] W. Zelazko, "A characterization of multiplicative linear functionals in complex Banach algebras," Studia Mathematica, vol. 30, pp. 83-85, 1968.

[5] A. Zivari-Kazempour, "A characterization of Jordan homomorphism on Banach algebras," Chinese Journal of Mathematics, Article ID 698621, 3 pages, 2014.

[6] A. Zivari-Kazempour, "A characterisation of 3-Jordan homomorphisms on Banach algebras," Bulletin of the Australian Mathematical Society, vol. 93, no. 2, pp. 301-306, 2016.

[7] M. Eshaghi Gordji, T. Karimi, and S. Kaboli Gharetapeh, "Approximately $n$-Jordan homomorphisms on Banach algebras," Journal of Inequalities and Applications, vol. 2009, Article ID 870843, 8 pages, 2009.

[8] G. An, Y. Ding, and J. Li, "Characterizations of Jordan left derivations on some algebras," Banach Journal of Mathematical Analysis, vol. 10, no. 3, pp. 466-481, 2016.

[9] L. Liu, "On Jordan centralizers of triangular algebras," Banach Journal of Mathematical Analysis, vol. 10, no. 2, pp. 223-234, 2016.

[10] A. Sahleh and A. Zivari-Kazempour, "Arens regularity of certain class of Banach algebras," Abstract and Applied Analysis, vol. 2011, Article ID 680952, 6 pages, 2011.

[11] H. G. Dales, Banach Algebras and Automatic Continuity, vol. 24 of London Mathematical Society Monographs, The Clarendon Press, Oxford, UK, 2000.
[12] H. G. Dales and A. T. M. Lau, "The second duals of Beurling algebras," Memoirs of the American Mathematical Society, vol. 177, no. 836, pp. 1-199, 2005.

[13] N. Dunford and J. T. Schwartz, Linear Operators, Part I, WileyInterscience, New York, NY, USA, 1958.

[14] F. Ghahramani, R. J. Loy, and G. A. Willis, "Amenability and weak amenability of second conjugate Banach algebras," Proceedings of the American Mathematical Society, vol. 124, no. 5, pp. 1489-1497, 1996.

[15] J. A. Baker, "The stability of the cosine equation," Proceedings of the American Mathematical Society, vol. 80, no. 3, pp. 411-416, 1980. 


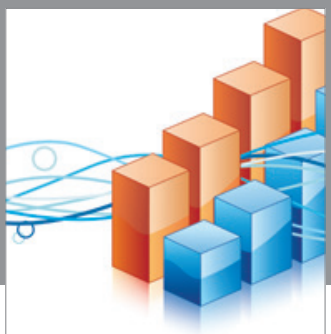

Advances in

Operations Research

vatem alat4

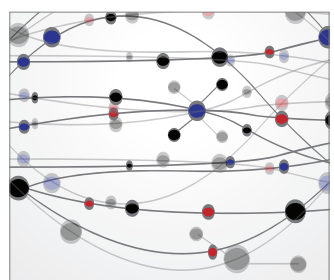

\section{The Scientific} World Journal
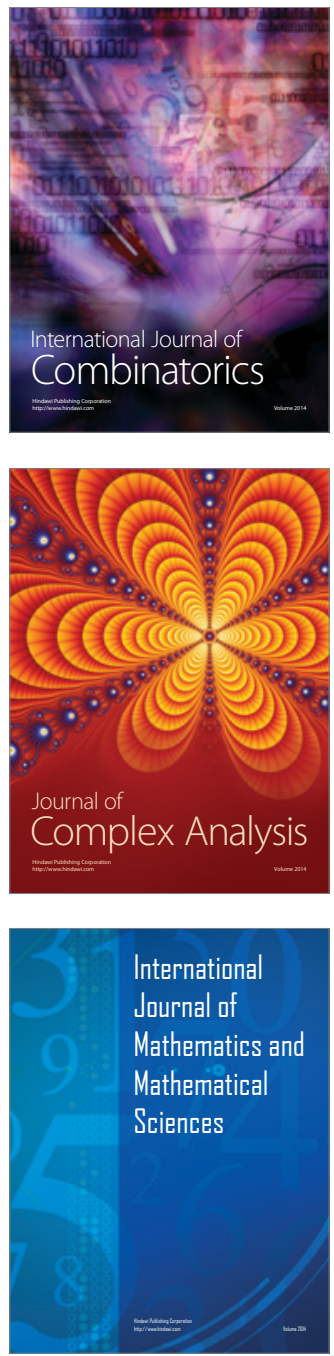
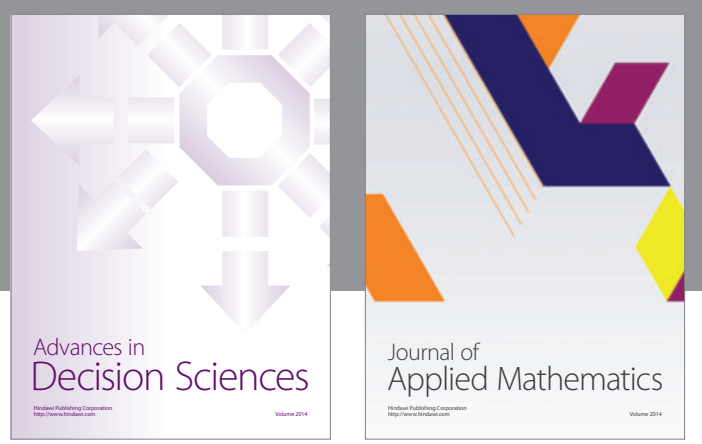

Algebra

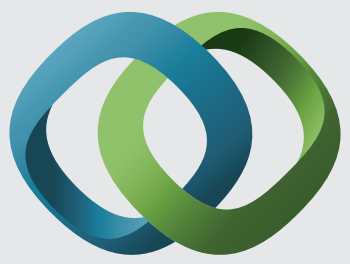

\section{Hindawi}

Submit your manuscripts at

https://www.hindawi.com
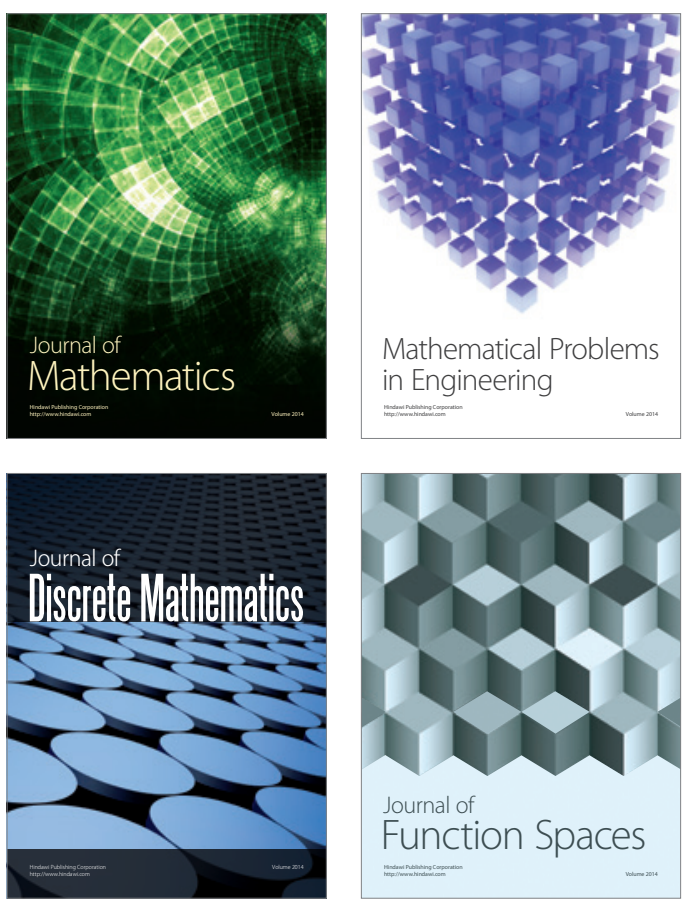

Mathematical Problems in Engineering
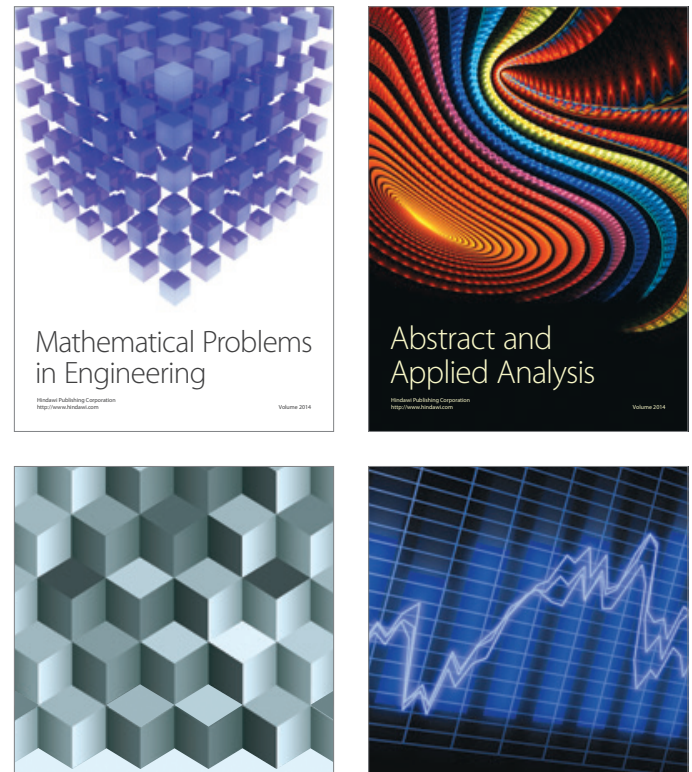

Journal of

Function Spaces

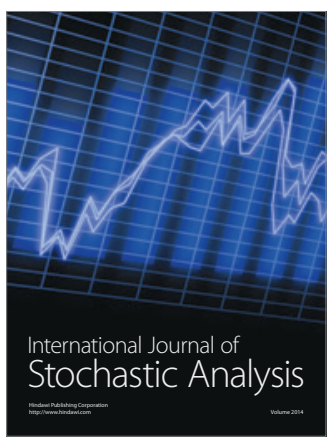

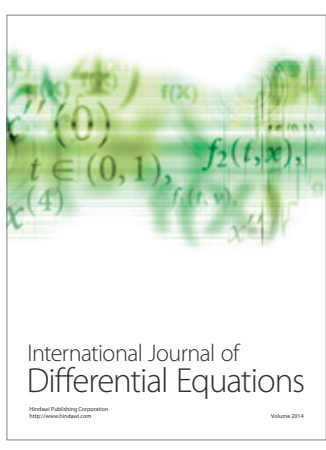
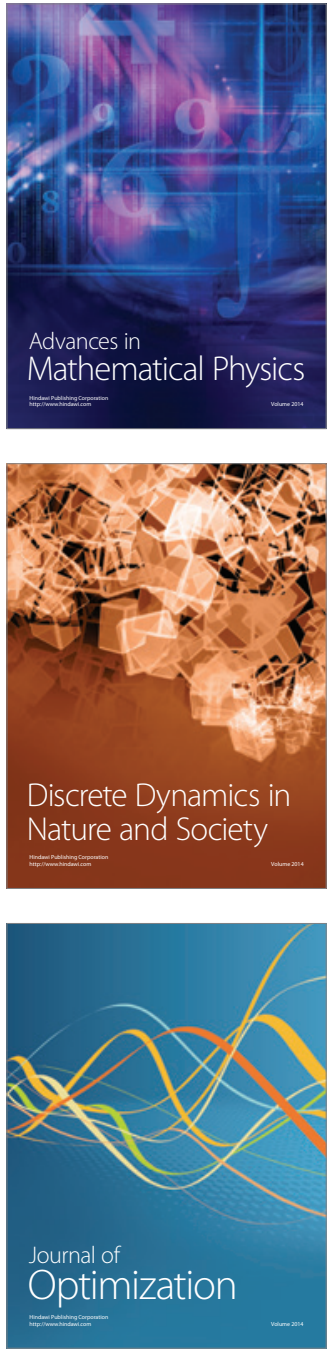